Article

\title{
Conflicting Information Fusion Based on an Improved DS Combination Method
}

\author{
Jie Chen, Fang Ye, Tao Jiang and Yuan Tian * \\ College of Information and Communication Engineering, Harbin Engineering University, Harbin 150001, China; \\ sandra@hrbeu.edu.com (J.C.); yefang0923@126.com (F.Y.); jiangtao_heu@126.com (T.J.) \\ * Correspondence: tianyuan347@126.com; Tel.:+86-188-4617-9039
}

Received: 30 October 2017; Accepted: 15 November 2017; Published: 16 November 2017

\begin{abstract}
An effective and reliable fusion method for conflicting information is proposed in this paper. Compared with a single-sensor system, a multi-sensor fusion system can comprehensively combine the redundancy and complementarity of multi-sensor information to obtain better system performance. Hence, the multi-sensor fusion system has become one of the research hotspots. However, due to lack knowledge about the measurement environment and limited sensor accuracy, the multi-sensor system inevitably appears to have imperfect, uncertain and inconsistent information. To solve the problem, we introduce one powerful uncertainty reasoning method: Dempster-Shafer theory (DS theory). With convincing measurement and a forceful combination of uncertain information, DS theory is widely applied in various fields, like decision-making, expert systems, target tracking, monitoring systems, etc. Nevertheless, DS theory will produce counter-intuitive fusion results when the pieces of evidence are highly conflicting. To address this issue, we raise an improved DS combination method for conflicting information fusion in this paper. First of all, the modified Minkowski distance function and the betting-commitment distance function are separately employed to revise potentially conflicting pieces of evidence. The procedure availably solves the conflicting situations caused by unreliable and imprecise evidence sources, which enhances the consistency among pieces of evidence. Then, based on two revised pieces of evidence, a conflicting redistribution strategy based on locally conflicting analyses is put forward. The approach dexterously combines two revised pieces of evidence to avoid conflicting situations caused by compulsive normalization, which further improves the accuracy and convergence speed of the multi-sensor fusion system. Finally, two experimental analyses with consistent information and conflicting information reveal the remarkable effectiveness and priority of the proposed algorithm for the multi-sensor fusion system. Consequently, this paper has certain value for the multi-sensor fusion system.
\end{abstract}

Keywords: multi-sensor fusion; uncertain information; Dempster-Shafer theory; conflicting pieces of evidence

\section{Introduction}

The the multi-sensor system has attracted much attention since data from a single sensor are often limited and unreliable [1,2]. Information fusion technology is a typical information processing method for the multi-sensor system, which has become an inexorable trend in various military and civilian areas. With the rapid rise and wide application of sensor technology and computer science, multi-sensor information fusion technology has become an emerging and significant subject. The multi-sensor information fusion algorithm is able to integrate the redundant and complementary information of different sensors, which can obtain better decision-making performance [3,4].

However, because of different sensor sensitivities in the multi-sensor system and various potential deception and interference in a hostile environment, the multi-sensor information provided by multi-sensor sources is normally imperfect, uncertain and inconsistent $[5,6]$. 
How to synthesize this uncertain information to get accurate fusion results is the focus in the multi-sensor system. Evidence theory, also called Dempster-Shafer theory (DS theory), is a widely-used uncertain reasoning method $[7,8]$. DS theory can not only fuse the complementary information to reduce the system uncertainty, but also fuse the redundant information to enhance the system stability $[9,10]$. Further, DS theory can produce reasonable decision-making results even with partially erroneous or incorrect sensor data, which strengthens system robustness [11].

Nevertheless, the enormous uncertainty that is caused by a complex monitoring environment (bad weather, dense noise, man-made interference, etc.) and possibly inconsistent data (poor anti-interference ability of sensors, incomplete or imprecise sensor processing, etc.) consequentially leads to high conflicts in the multi-sensor fusion system. With high evidence conflicts, DS theory will get counter-intuitive results [12]. This phenomenon that the combination of the results of conflicting pieces of evidence will be opposite of common sense is defined as a paradox. Since these fusion paradoxes were put forward by Zadeh [13], the establishment of a rational and effective multi-sensor fusion method has become one of the most significant aspects in the research of DS theory.

Some researchers believe that the evidence conflicts are mainly caused by the peremptory normalization step of the DS combination rule in DS theory. In the DS combination rule, to guarantee the normalization property of fusion results, the normalization step directly deals with the partial conflicts with global processing. This unreasonable management may lead to counter-intuitive fusion results. To address the issue, Smets and Yager [14,15] optimize the DS combination rule through different processing means. With different understandings of the completeness of the frame of discernment $\Theta$, Smets and Yager [14,15] separately assign the whole conflicting mass to $\varnothing$ and $\Theta$. Theoretically, both methods solve the conflicting problem and ensure the normalization property of fusion results; while practically, the system uncertainty still exists. Instead of blindly denying the conflicting information, Sun et al. [16] firstly classified pieces of evidence into consistent pieces of evidence and inconsistent pieces of evidence-based conflicting analyses and then used a novel redistribution formula to solve the conflicting situations. Besides, Li et al. [17] also affirmed the useful information of conflicting pieces of evidence and put forward a weighted conflicting redistribution principle. Moreover, from the perspective of locally-conflicting analyses, Zhang et al. [18,19] respectively propose two local conflict assignment methods. Unfortunately, both methods sometimes violate the theoretical properties of the DS combination rule like commutativity and associativity.

Other scholars regard the DS combination rule as a well-derived method, and the unreasonable fusion results are primarily caused by the uncertainty of sensor sources; where the erroneous uncertainty of sensor sources is brought in by the inconsistent performances of sensors, potential deception and interference in a hostile environment. To solve the problem, Murphy et al. [20,21] use different processing methods to modify pieces of evidence before combination. The works in $[20,21]$ separately revised the conflicting pieces of evidence with average processing and weighted processing, which aroused a neoteric study trend for solving conflicting situations. Accordingly, Deng [22] employed the Jousselme distance function to evaluate the credibility degrees of pieces of evidence and then raised a modified DS combination rule based on evidence credibility. To accelerate the convergence rate of fusion processing, an improved combination method based on absolute reliability is introduced in [23]. In addition, a conflicting information fusion based on the K-L (Kullback-Leibler) distance function is shown in [24], and a conflicting information fusion based on the Mahalanobis distance function is exhibited in [25]. However, the Mahalanobis distance function requires the covariance computation of the matrix, which is not suitable for large-scale data processing.

From my point of view, both the above standpoints about evidence conflicts are practicable and reasonable. The DS combination rule does have a conflicting possibility due to the straightforward normalization step, and the inevitable uncertainty of multi-sensor information is also a conflicting source worth thinking about. Therefore, we comprehensively combine these two conflict processing methods to improve the rationality and effectiveness of fusion results. The comprehensive processing 
of evidence conflicts will promote a greater application prospect for the multi-sensor conflicting information fusion based on DS theory.

In this paper, we present an improved DS combination method for conflicting information fusion. The proposed algorithm is mainly accomplished by two steps. First and foremost, to measure and eliminate the conflicting situation caused by potential interference of the environment and limited accuracy of sensors, the modified Minkowski distance function and the betting-commitment distance function are separately employed to revise the pieces of evidence in the multi-sensor fusion system. After that, based on locally conflicting analyses of two revised pieces of evidence, an improved conflicting redistribution strategy is raised to eradicate the conflicting possibility caused by blunt normalization. Applying the comprehensive management of conflicting pieces of evidence, the proposed algorithm will obtain rational and precise fusion results for the multi-sensor system.

The rest of this paper is structured as follows. Section 2 gives a brief review of DS theory, which is the mathematical foundation of this paper. Limitations of conventional DS theory are presented in Section 3, followed by the introduction of two typically modified DS combination methods in Section 4 . As the innovation of this paper, Section 5 emphasizes the conflicting information fusion algorithm based on the improved DS combination method. Section 6 analyzes and evaluates the performance of the proposed algorithm through two numerical experiments. Finally, concluding remarks are offered in the last section.

\section{Review of DS Theory}

DS theory is an effective fusion method for uncertain information. DS theory can handle imprecise and imperfect information with its unique probability quantifications and valid uncertainty measurement. Thus, compared with probability theory, DS theory has broader application areas, like expert systems [26,27], decision-making [28,29], fault diagnosis [30,31], target tracking [32], image restorations [33], etc.

\subsection{Frame of Discernment}

To apply the multi-sensor fusion method based on DS theory, we firstly introduce a set of hypotheses called the frame of discernment (frame); where hypotheses represent all the possible results that people can recognize for the multi-sensor information fusion processing. In this paper, the frame is defined to contain $M$ mutually exclusive and exhaustive hypotheses.

$$
\Theta=\left\{H_{1}, H_{2}, \cdots, H_{M}\right\}
$$

where $H_{i}$ represents the $i$-th hypothesis in the multi-sensor fusion system.

Then, the power set $2^{\Theta}$ is derived.

$$
2^{\Theta}=\left\{\varnothing,\left\{H_{1}\right\},\left\{H_{2}\right\}, \cdots,\left\{H_{M}\right\},\left\{H_{1}, H_{2}\right\}, \cdots,\left\{H_{1}, H_{M}\right\}, \cdots,\left\{H_{1}, H_{2}, \cdots, H_{M}\right\}\right\}
$$

where $\varnothing$ is the empty set and each element of the power set $2^{\Theta}$ is named as a proposition of the multi-sensor fusion system.

It is obvious that $2^{\Theta}$ is composed of $2^{M}$ propositions, and any proposition $A \subseteq \Theta$ meets $A \in 2^{\Theta}$.

\subsection{Mass Function}

The basis of DS theory is the mass function (mass), also called basic probability assignment or basic belief assignment. Mass is a function $m: 2^{\Theta} \rightarrow[0,1]$, that satisfies the following equations.

$$
\begin{gathered}
m(\varnothing)=0 \\
\sum_{A \subseteq \Theta} m(A)=1
\end{gathered}
$$


for proposition $A \in 2^{\Theta}$; where mass function $m(A)$ expresses the basic support degree of evidence $m$ to proposition $A$.

Clearly, Equation (3) reflects the non-negativity of mass, and Equation (4) ensures the normalization property of mass. Due to the lack of further knowledge, $m(A)$ cannot be subdivided into its proper subsets. Besides, if $m(A)>0, H$ is called the focal element, and all the focal elements constitute the mass core.

\subsection{Uncertainty Quantization}

Since mass $m(A)$ is presented, we then introduce two functions: belief function (Bel) and plausibility function $(\mathrm{Pl})$ on $2^{\Theta}$.

$$
\begin{aligned}
& \operatorname{Bel}(A)=\sum_{B \subseteq A} m(B) \\
& \operatorname{Pl}(A)=\sum_{B \cap A \neq \varnothing} m(B)
\end{aligned}
$$

It is easy to check in Equations (5) and (6) that $\operatorname{Bel}(A)$ reports the possibility that mass $m$ totally supports proposition $A$, while $P l(A)$ describes the possibility that mass $m$ does not regard proposition $A$ as false. According to Equations (5) and (6), it follows the relationship of $\operatorname{Bel}(A)$ and $\operatorname{Pl}(A)$.

$$
\begin{gathered}
\operatorname{Bel}(A) \leq \operatorname{Pl}(A) \\
\operatorname{Pl}(A)=1-\operatorname{Bel}(\bar{A})
\end{gathered}
$$

where $\bar{A}$ is the complement set of $A$.

As is known, $\operatorname{Bel}(\bar{A})$ is the doubt function opposite of $\operatorname{Bel}(A)$, which reflects the doubt degree of mass $m$ to proposition $A$ (details can be seen in [7,8]). Thus, $P l(A)$ in Equation (8) reflects the unsuspicious degree of proposition $A$, which conforms to its definition in Equation (6). Obviously, $\operatorname{Bel}(A)$ and $\operatorname{Pl}(A)$ respectively denote the upper and lower bounds of probability, which form the uncertainty interval $[\operatorname{Bel}(A), \operatorname{Pl}(A)]$ (shown in Figure 1).

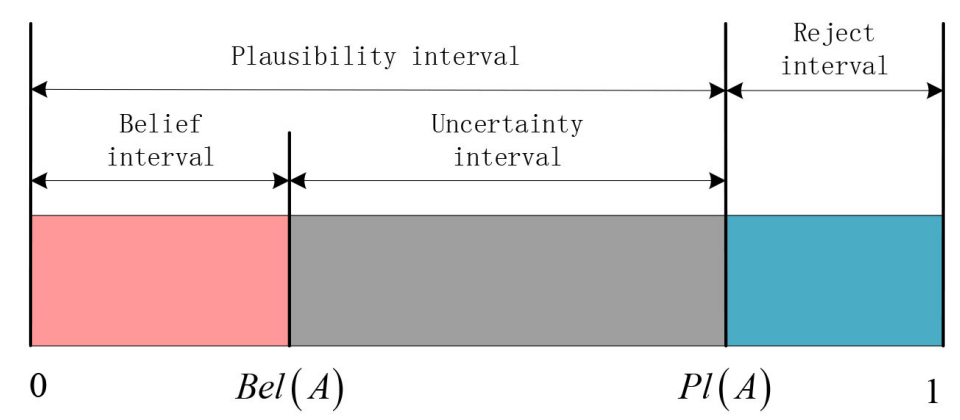

Figure 1. Uncertainty description of proposition $A$.

\subsection{DS Combination Rule}

The core to build a multi-sensor fusion system based on DS theory is the DS combination rule. Assume that the multi-sensor's frame is $\Theta=\left\{H_{1}, H_{2}, \cdots, H_{M}\right\}$, and there are two pieces of evidence $m_{1}, m_{2}$ obtained from two independent sensors. The DS combination rule can be defined as:

$$
m(H)= \begin{cases}\frac{1}{1-K} \sum_{H_{i} \cap H_{j}=H} m_{1}\left(H_{i}\right) \cdot m_{2}\left(H_{j}\right) & \text { if } \quad H \neq \varnothing \\ 0 & \text { if } \quad H=\varnothing\end{cases}
$$


where $K$ is the conflicting factor that reflects the conflicting degree of pieces of evidence $m_{1}, m_{2}$ and $\frac{1}{1-K}$ is the normalized factor that makes the fusion results meet the normalization property in Equation (4).

$$
K=\sum_{H_{i} \cap H_{j}=\varnothing} m_{1}\left(H_{i}\right) \cdot m_{2}\left(H_{j}\right)
$$

Clearly, the DS combination rule directly sums pieces of evidence $m_{1}, m_{2}$. Thus, it is also called the direct sum operation, expressed as $m=m_{1} \oplus m_{2}$. Figure 2 intuitively describes the combined processing of pieces of evidence $m_{1}, m_{2}$.

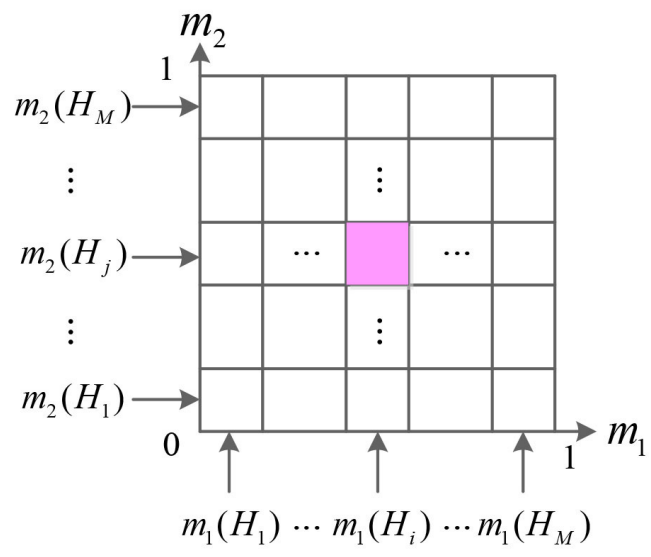

Figure 2. Diagrammatic sketch of the combination of pieces of evidence $m_{1}, m_{2}$.

Apparently, the DS combination rule in Equation (9) satisfies both the commutative law and associative law.

$$
\begin{aligned}
m_{1} \oplus m_{2} & =m_{2} \oplus m_{1} \\
\left(m_{1} \oplus m_{2}\right) \oplus m_{3} & =m_{1} \oplus\left(m_{2} \oplus m_{3}\right)
\end{aligned}
$$

For multi-sensor pieces of evidence $m_{1}, m_{2}, \cdots, m_{N}$, the DS combination rule is extended as:

$$
m(H)=\left\{\begin{array}{lll}
\frac{1}{1-K} \sum_{\cap H_{j}=H, j=1,2, \cdots, M} \prod_{1 \leq i \leq N} m_{i}\left(H_{j}\right) & \text { if } & H \neq \varnothing \\
0 & \text { if } & H=\varnothing
\end{array}\right.
$$

where the conflicting degree $K$ of $N$ pieces of evidence is:

$$
K=\sum_{\cap H_{j}=\varnothing, j=1,2, \cdots, M} \prod_{1 \leq i \leq N} m_{i}\left(H_{j}\right)
$$

\section{Limitations of Traditional DS Theory}

On the one hand, Equation (9) will produce counter-intuitive results with a high conflicting factor. What is worse is that Equation (9) cannot be employed if the conflicting factor $K=1$ (in this situation, the denominator of the normalized factor $\frac{1}{1-K}$ is zero). Evidently, the mandatory normalization step of the DS combination rule limits the application of DS theory to some extent. On the other hand, due to the effect of background noises and man-made interference, multi-sensor information always has inconsistent reliability degrees. The inconsistency among multi-sensor sources indirectly causes evidence conflicts, which are hard to handle by traditional DS evidence theory.

To better demonstrate the applicable limitations of DS theory, we give three conflicting examples. 
Example 1. Suppose that the frame of the multi-sensor fusion system is $\Theta=\{A, B, C\}$ and there are two pieces of evidence processed in the multi-sensor system.

$$
\left\{\begin{array}{l}
E_{1}: m_{1}(A)=0.99, m_{1}(B)=0.01, m_{1}(C)=0 \\
E_{2}: m_{2}(A)=0, m_{2}(B)=0.01, m_{2}(C)=0.99
\end{array}\right.
$$

We can see from Equation (15) that pieces of evidence $E_{1}, E_{2}$ are highly conflicting. Separately using Equations (9) and (10), we can get the fusion results and conflicting factor.

$$
\begin{gathered}
m(A)=0, m(B)=1, m(C)=0 \\
K=0.9999
\end{gathered}
$$

Surprisingly, as pieces of evidence $E_{1}, E_{2}$ both support proposition $B$ with minimum probability, the fusion results unexpectedly believe that proposition $B$ is true. This paradoxical situation is caused by the highly conflicting degree of pieces of evidence $E_{1}, E_{2}$ shown in Equation (17).

Example 2. Suppose that the frame of the multi-sensor fusion system is $\Theta=\{A, B, C\}$ and there are four pieces of evidence processed in the multi-sensor system.

$$
\left\{\begin{array}{l}
E_{1}: m_{1}(A)=0.7, m_{1}(B)=0.2, m_{1}(C)=0.1 \\
E_{2}: m_{2}(A)=0, m_{2}(B)=0.9, m_{2}(C)=0.1 \\
E_{3}: m_{3}(A)=0.75, m_{3}(B)=0.15, m_{3}(C)=0.1 \\
E_{4}: m_{4}(A)=0.8, m_{4}(B)=0.1, m_{4}(C)=0.1
\end{array}\right.
$$

In Equation (18), pieces of evidence $E_{1}, E_{3}, E_{4}$ are relatively consistent, and they all assign proposition $A$ with the biggest support. While evidence $E_{2}$ is inconsistent with the above-mentioned pieces of evidence $E_{1}, E_{3}, E_{4}$, it completely denies proposition $A$. It is intuitive that fusion results should be close to consistent pieces of evidence $E_{1}, E_{3}, E_{4}$. That is, the fusion results should allocate proposition $A$ with the biggest support.

Separately using Equations (13) and (14), we can get the fusion results and conflicting factor.

$$
\begin{gathered}
m(A)=0, m(B)=0.9643, m(C)=0.0357 \\
K=0.99
\end{gathered}
$$

It is clear in Equation (19) that the fusion results using the DS combination rule are opposite of common sense. The inconsistency of single evidence $E_{2}: m_{2}(A)=0$ leads to the irrationality of all the fusion results. Obviously, this kind of count-intuitive phenomenon, called the "one ballot veto" paradox, is caused by the inconsistent evidence $E_{2}$.

Example 3. Suppose that the frame of the multi-sensor fusion system is $\Theta=\{A, B, C, D, E\}$ and there are five pieces of evidence processed in the multi-sensor system.

$$
\left\{\begin{array}{l}
E_{1}: m_{1}(A)=0.7, m_{1}(B)=0.1, m_{1}(C)=0.1, m_{1}(D)=0, m_{1}(E)=0.1 \\
E_{2}: m_{2}(A)=0, m_{2}(B)=0.5, m_{2}(C)=0.2, m_{2}(D)=0.1, m_{2}(E)=0.2 \\
E_{3}: m_{3}(A)=0.6, m_{3}(B)=0.1, m_{3}(C)=0.15, m_{3}(D)=0, m_{3}(E)=0.15 \\
E_{4}: m_{4}(A)=0.55, m_{4}(B)=0.1, m_{4}(C)=0.1, m_{4}(D)=0.15, m_{4}(E)=0.1 \\
E_{5}: m_{5}(A)=0.6, m_{5}(B)=0.1, m_{5}(C)=0.2, m_{5}(D)=0, m_{5}(E)=0.1
\end{array}\right.
$$

Separately using Equations (13) and (14), we can get the fusion results and conflicting factor.

$$
m(A)=0, m(B)=0.5556, m(C)=0.2222, m(D)=0, m(E)=0.2222
$$




$$
K=0.9999
$$

Recalling the analyses in Example 2, the fusion results in Equation (22) are apparently unreasonable. In the multi-sensor fusion system, consistent pieces of evidence $E_{1}, E_{3}, E_{4}, E_{5}$ all support proposition $A$ with the biggest probability. Thus, reliable fusion results should assign proposition $A$ the biggest support. However, the emergence of conflicting evidence $E_{2}$ directly leads to the wrong results of the whole system; where fusion results totally deny proposition $A$.

These perverse phenomena discussed illustrate the applicable limitations of DS theory. From the above descriptions, we can conclude that conflicting situations generally exist in the multi-sensor fusion system. At this point, the DS combination rule cannot achieve accurate multi-sensor fusion. Therefore, the core of the multi-sensor fusion system is to build a conflicting information fusion method through the improvement of DS theory.

\section{Existing Modified Methods}

As mentioned in Section 1, the processing modes of evidence conflicts are mainly divided into two categories. In this paper, we adopt two existing modified methods to illustrate the features of two processing modes of evidence conflicts.

\subsection{Modified Combination Method Based on Locally-Conflicting Analyses}

The first kind of processing method for evidence conflicts is based on the correction of the DS combination rule. It firstly cancels the normalization step and then realizes reasonable fusion method via effective assignment of evidence conflicts. One of the most typical methods is the modified combination method based on locally-conflicting analyses (details seen in [16]).

Suppose that there are $N$ pieces of evidence processed in the multi-sensor system. The modified combination method in [16] is shown as:

$$
m(H)=\left\{\begin{array}{lc}
p(H)+K \cdot \varepsilon \cdot q(H) & \text { if } H \neq \varnothing, \Theta \\
p(\Theta)+K \cdot \varepsilon \cdot q(\Theta)+K \cdot(1-\varepsilon) & \text { if } H=\Theta \\
0 & \text { if } H=\varnothing
\end{array}\right.
$$

where $K$ is the conflicting factor of $N$ pieces of evidence, $\varepsilon$ is the evidence credibility based on locally-conflicting analyses and $p(H), q(H)$ are two auxiliary functions.

$$
\begin{gathered}
\varepsilon=e^{-\bar{K}} \\
p(H)=\sum_{H_{1} \cap H_{2} \cap \cdots \cap H_{N}=H} m_{1}\left(H_{1}\right) m_{2}\left(H_{2}\right) \cdots m_{N}\left(H_{N}\right) \\
q(H)=\frac{1}{N} \sum_{i=1}^{N} m_{i}(H)
\end{gathered}
$$

where $\bar{K}$ represents the average conflicting degree of all locally-conflicting degrees $k_{i j}(i, j=1,2, \cdots, N)$.

$$
\begin{aligned}
\bar{K} & =\frac{1}{N(N-1) / 2} \sum_{i<j \leq N} k_{i j} \\
k_{i j} & =\sum_{H_{i} \cap H_{j}=\varnothing} m_{i}\left(H_{i}\right) m_{j}\left(H_{j}\right)
\end{aligned}
$$

The characteristic of [16] is that locally-conflicting analyses instead of globally-conflicting analyses are employed to accurately describe the evidence conflicting degree. Thus, the fusion results are more reasonable and reliable. 


\subsection{Revised Evidence Method Based on the Mahalanobis Distance Function}

Another kind of processing method for evidence conflicts is based on the revision of original pieces of evidence before combination. Before using the DS combination rule, it quantifies the evidence conflicts and next modifies pieces of evidence by analyzing the differences among pieces of evidence. One of the most typical methods is the revised pieces of evidence method based on Mahalanobis distance function (details seen in [25]).

Suppose that there are $N$ pieces of evidence processed in the multi-sensor system. The revised pieces of evidence method based on the Mahalanobis distance function in [25] is shown as:

$$
M_{k}\left(H_{i}\right)= \begin{cases}\omega_{k} \cdot m_{k}\left(H_{i}\right) & \text { if } H_{i} \neq \Theta \\ 1-\sum_{H_{i} \neq \varnothing} \omega_{k} \cdot m_{k}\left(H_{i}\right) & \text { if } H_{i}=\Theta\end{cases}
$$

where $\omega_{k}(k=1,2, \cdots, N)$ is the credibility degree of evidence $m_{k}$, which satisfies:

$$
\sum_{k=1}^{N} \omega_{k}=1
$$

The calculation of evidence credibility $\omega_{k}$ is shown below.

Step 1: Introduction of the Mahalanobis distance function. The Mahalanobis distance function between pieces of evidence $m_{1}, m_{2}$ is defined as:

$$
d_{12}=d_{M}\left(m_{1}, m_{2}\right)=\left(m_{1}-m_{2}\right)^{T} \Sigma^{-1}\left(m_{1}-m_{2}\right)
$$

where $\Sigma$ is the covariance matrix of evidence vectors $\mathbf{M}$.

$$
\mathbf{M}=\left[\begin{array}{llll}
m_{1}\left(H_{1}\right) & m_{1}\left(H_{2}\right) & \cdots & m_{1}\left(H_{M}\right) \\
m_{2}\left(H_{1}\right) & m_{2}\left(H_{2}\right) & \cdots & m_{2}\left(H_{M}\right)
\end{array}\right]
$$

Step 2: Quantization about differences among pieces of evidence. After the calculation of the Mahalanobis distance function, the distance matrix can be defined.

$$
\mathbf{D}=\left[\begin{array}{cccc}
0 & d_{12} & \cdots & d_{1 N} \\
d_{21} & 0 & \cdots & d_{2 N} \\
\vdots & \vdots & & \vdots \\
d_{N 1} & d_{N 2} & \cdots & 0
\end{array}\right]
$$

Besides, the average distance of evidence $m_{i}$ to other pieces of evidence can be obtained.

$$
\bar{d}_{i}=\frac{\sum_{j=1}^{n} d_{i j}}{n-1}
$$

Step 3: Establishment of evidence credibility $\omega_{k}$. Through normalizing the average distance, the weight of every piece of evidence is calculated.

$$
\omega_{k}=\frac{\max _{1 \leq i \leq n}\left(\bar{d}_{i}\right)}{\bar{d}_{k}}
$$

Apparently, the calculated evidence credibility $\omega_{k}$ meets the normalization property in Equation (31).

The characteristic of Lin et al. [25] is that this method takes full account of the difference and reliability of evidence sources and ulteriorly makes relevant revisions to pieces of evidence before 
combination. Then, since the Mahalanobis distance has nothing to do with units, there is no need to preprocess, which greatly simplifies the implementation steps of the fusion processing.

\section{Improved DS Combination Method}

In order to weaken the negative impact of inconsistent multi-sensor information and simultaneously enhance the robustness of the multi-sensor fusion system, pieces of evidence starve for precise evaluation of their reliabilities and further modifications.

Accordingly, the improved DS combination method proposed in this paper firstly introduces the modified Minkowski distance function and the betting-commitment distance function to respectively evaluate evidence conflicts and revise the potentially conflicting pieces of evidence. Then, an original conflicting redistribution strategy based on locally-conflicting analyses is raised to further solve the conflicting situations. The flow diagram of the proposed DS combination method is shown is Figure 3.

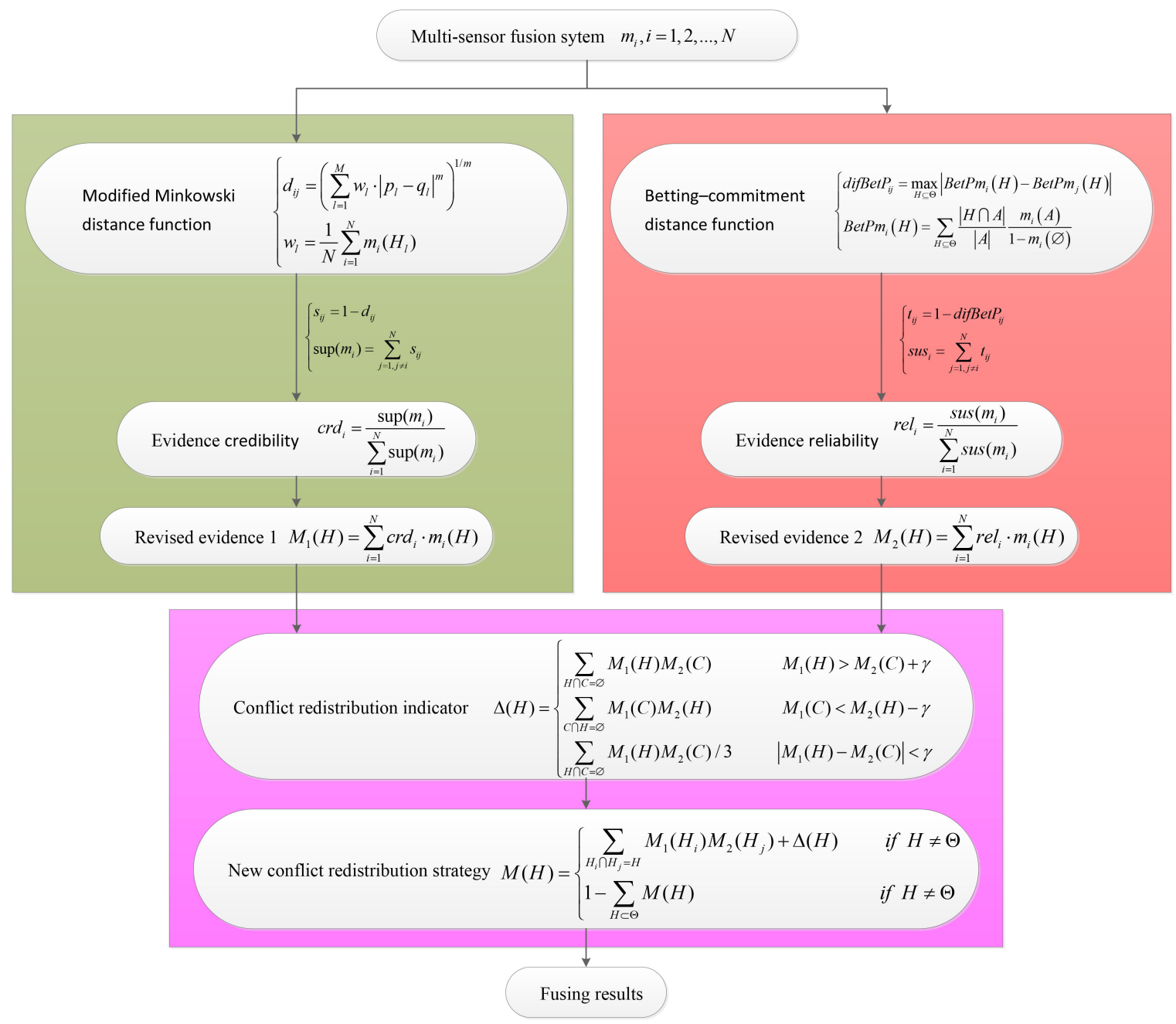

Figure 3. Implementation block of the proposed algorithm.

In Figure 3, the green rectangle, peach rectangle and purple rectangle, respectively, represent the revision processing of pieces of evidence based on the modified Minkowski distance function, the revision processing of pieces of evidence based on the betting-commitment distance function and the original conflicting redistribution strategy based on locally-conflicting analyses. Their specific implementation steps are shown below. 


\subsection{Two Revised Pieces of Evidence Based on Different Distance Functions}

The fusion results in [22-25] reflect that different distance functions have different measurements for conflicting pieces of evidence. Additionally, the rationality and reliability of fusion results in [22-25] testify that adopting a suitable distance function will effectively handle conflicting situations. Thus, we separately propose the modified Minkowski distance function and the betting-commitment distance function.

\subsubsection{Revised Evidence Based on the Modified Minkowski Distance Function}

Step 1: Modification of the Minkowski distance function.

Suppose that the frame of the multi-sensor fusion system is $\Theta=\left\{H_{1}, H_{2}, \cdots, H_{M}\right\}$ and there are $N$ pieces of evidence processed in the multi-sensor system.

$$
E_{i}: m_{i}(H)=\left\{\begin{array}{lll}
p_{1} & & H=H_{1} \\
p_{2} & & H=H_{2} \\
& \ldots & \\
p_{M} & H=H_{M}
\end{array} \quad E_{j}: m_{j}(H)=\left\{\begin{array}{lll}
q_{1} & & H=H_{1} \\
q_{2} & & H=H_{2} \\
& \ldots & \\
q_{M} & & H=H_{M}
\end{array}\right.\right.
$$

where $i, j=1,2, \cdots, N$ are the evidence indexes.

The Minkowski distance function is a distance measurement in European space. It is an extension of the Euclidean distance function and Manhattan distance function. According to the introduction of the Minkowski distance function [34], the distance function $d_{\min }\left(m_{i}, m_{j}\right)$ of $m_{i}$ and $m_{j}$ can be derived.

$$
d_{\min }\left(m_{i}, m_{j}\right)=\left(\sum_{l=1}^{M}\left|p_{l}-q_{l}\right|^{m}\right)^{1 / m}
$$

where $m$ is the measurement index.

In order to accelerate the convergence speed, we propose a weighting index $w_{l}(l=1,2, \cdots, M)$ to modify the Minkowski distance function.

$$
\begin{aligned}
d_{i j}=d\left(m_{i}, m_{j}\right) & =\left(\sum_{l=1}^{M} w_{l} \cdot\left|p_{l}-q_{l}\right|^{m}\right)^{1 / m} \\
w_{l} & =\frac{1}{N} \sum_{i=1}^{N} m_{i}\left(H_{l}\right)
\end{aligned}
$$

Since $w_{l}$ is the average mass of all pieces of evidence to proposition $H_{l}$, it indirectly expresses the importance of proposition $H_{l}$. Hence, the modified Minkowski distance function in Equation (39) can better distinguish the credibility degrees of different pieces of evidence.

Step 2: Calculation of evidence credibility.

To describe the relationship of $N$ pieces of evidence, we then calculate their similarity degrees $s_{i j}$.

$$
s_{i j}=1-d_{i j}
$$

As is clearly seen in Equation (41), $s_{i j}$ is inversely proportional to $d_{i j}$. That is, the more different pieces of evidence $E_{i}, E_{j}$ there are, the less similar they are, and vice versa.

Accordingly, we can derive the pieces of evidence's support degrees sup $p_{i}$ and credibility degrees $\mathrm{crd}_{i}$.

$$
\sup _{i}=\sum_{j=1, j \neq i}^{N} s_{i j}
$$




$$
\operatorname{crd}_{i}=\frac{\sup _{i}}{\sum_{i=1}^{N} s u p_{i}}
$$

Equation (42) reveals that $\sup _{i}$ reflects the total similarity of evidence $m_{i}$ with other pieces of evidence. After normalizing sup ${ }_{i}, \mathrm{crd}_{i}$ in Equation (43) reports the evidence's credibility compared with other pieces of evidence. Clearly, $\mathrm{crd}_{i}$ satisfies the normalization property.

$$
\sum_{i=1}^{N} \operatorname{crd}_{i}=1
$$

Step 3: Revision of pieces of evidence based on evidence credibility.

Based on the credibility degrees $c r d_{i}$ discussed above, we apply the following equation to revise the pieces of evidence.

$$
M_{1}(H)=\sum_{i=1}^{N} c r d_{i} \cdot m_{i}(H)
$$

\subsubsection{Revised Evidence Based on the Betting-Commitment Distance Function}

Similar to the above processing, revised evidence based on the betting-commitment distance function is calculated by three steps.

Step 1: Introduction of the betting-commitment distance function.

Under the multi-sensor fusion system with $N$ pieces of evidence, we introduce the pignistic transformation and ulteriorly present the betting-commitment distance function $[35,36]$.

$$
\begin{gathered}
\operatorname{BetP}_{i}(H)=\sum_{H \subseteq \Theta} \frac{|H \cap A|}{|A|} \frac{m_{i}(A)}{1-m_{i}(\varnothing)} \\
\operatorname{difBetP} P_{i j}=\max _{H \subseteq \Theta}\left|\operatorname{BetP} m_{i}(H)-\operatorname{BetPm}_{j}(H)\right|
\end{gathered}
$$

for propositions $H, A \in 2^{\Theta}$; where $|A|$ is the cardinality of proposition $A$.

Step 2: Calculation of evidence reliability.

Then, we separately define the tolerance degrees $t_{i j}$, sustaining degrees $s u s_{i}$ and reliability degrees $\mathrm{rel}_{i}$ of $\mathrm{N}$ pieces of evidence.

$$
\begin{gathered}
t_{i j}=1-\operatorname{difBet} P_{i j} \\
\text { sus }_{i}=\sum_{j=1, j \neq i}^{N} t_{i j} \\
\text { rel }_{i}=\frac{\text { sus }_{i}}{\sum_{i=1}^{N} \text { sus }_{i}}
\end{gathered}
$$

Recalling the discussion of $\mathrm{crd}_{i}, r e l_{i}$ reports the reliability of evidence $m_{i}$, which satisfies the normalization property.

Step 3: Revision of pieces of evidence based on evidence reliability.

Employing the reliability degrees $r e l_{i}$ discussed above, the revised evidence can be obtained.

$$
M_{2}(H)=\sum_{i=1}^{N} r e l_{i} \cdot m_{i}(H)
$$

Through the comprehensive evaluation of conflicting pieces of evidence, these two revised pieces of evidence $M_{1}, M_{2}$ in Equations (45) and (51) evaluate and correct the potentially conflicting pieces of evidence from different angles. $M_{1}, M_{2}$ essentially solve the system uncertainty caused by unreliable 
evidence sources and imprecise preprocessing, which is beneficial to the subsequent combination and further decision-making.

\subsection{Conflicting Redistribution Strategy Based on Locally-Conflicting Analyses}

Up to now, conflicting pieces of evidence have been effectively revised. The next step is to modify the DS combination. Referring to Zhang et al. [19], we raise a conflicting redistribution strategy based on locally-conflicting analyses.

$$
M(H)= \begin{cases}\sum_{H_{i} \cap H_{j}=H} M_{1}\left(H_{i}\right) M_{2}\left(H_{j}\right)+\Delta(H) & \text { if } H \neq \varnothing, \Theta \\ 1-\sum_{H \subset \Theta} M(H) & \text { if } H=\Theta \\ 0 & \text { if } H=\varnothing\end{cases}
$$

where $\Delta(H)$ is the locally-conflicting redistribution indicator.

$$
\Delta(H)=\left\{\begin{array}{lc}
\sum_{H \cap C=\varnothing} M_{1}(H) M_{2}(C & M_{1}(H)>M_{2}(C)+\gamma \\
\sum_{C \cap H=\varnothing} M_{1}(C) M_{2}(H) & M_{1}(C)<M_{2}(H)-\gamma \\
\sum_{H \cap C=\varnothing} M_{1}(H) M_{2}(C) / 3 & \left|M_{1}(H)-M_{2}(C)\right|<\gamma
\end{array}\right.
$$

for propositions $H, C \subseteq \Theta$; where $\gamma \in[0,1]$ is the decision threshold. The value of $\gamma$ can be set based on a priori knowledge, which is generally distributed in $[0.1,0.5]$. To ensure the unity of simulation in this paper, $\gamma=0.2$.

In Equation (53), the local conflicting degree between pieces of evidence $M_{1}(H), M_{2}(C)$ is assigned to the proposition with larger support if their numerical difference is larger than the threshold $\gamma$. When the different value between pieces of evidence $M_{1}(H), M_{2}(C)$ is less than the threshold $\gamma$, propositions $H, C, \Theta$ are regarded to bear the same responsibility for the conflict. That is, the residual mass $\sum_{H \cap C=\varnothing} M_{1}(H) M_{2}(C) / 3$ is one average allocated to propositions $H, C, \Theta$ if $\left|M_{1}(H)-M_{2}(C)\right|<\gamma$.

Clearly, the local conflicting analyses of the proposed conflicting redistribution strategy conform to the intuitive judgment criterion. Compared with the global conflict allocation schemes, the local conflict analyses in Equations (52) and (53) enhance the rationality and availability of conflict assignment.

\section{Experimental Results and Analyses}

In this section, we use two simulation examples to illustrate the priority and effectiveness of the proposed algorithm. First of all, two multi-sensor data in [25] are adopted. The former one is the consistent information, and the other is the conflicting information. Secondly, some existing methods $[8,16,17,24,25]$ that were presented in Section 1, especially the two $[16,25]$ discussed in Section 4, are employed as the comparison methods.

\subsection{Multi-Sensor Fusion with Consistent Information}

Assume that the frame of the multi-sensor fusion system is $\Theta=\{A, B, C\}$ and there are five pieces of evidence processed in the multi-sensor system. Suppose that proposition $A$ is the true object; the consistent information of the multi-sensor system is shown in Table 1. 
Table 1. Mass functions of consistent information.

\begin{tabular}{cccc}
\hline \multirow{2}{*}{ Sensors } & \multicolumn{3}{c}{ Propositions } \\
\cline { 2 - 4 } & $\boldsymbol{A}$ & $\boldsymbol{B}$ & $\boldsymbol{C}$ \\
\hline$E_{1}: m_{1}(\cdot)$ & 0.90 & 0 & 0.10 \\
$E_{2}: m_{2}(\cdot)$ & 0.88 & 0.01 & 0.11 \\
$E_{3}: m_{3}(\cdot)$ & 0.50 & 0.20 & 0.30 \\
$E_{4}: m_{4}(\cdot)$ & 0.98 & 0.01 & 0.01 \\
$E_{5}: m_{5}(\cdot)$ & 0.90 & 0.05 & 0.05 \\
\hline
\end{tabular}

It can be seen in Table 1 that due to the complex environment and discrepant sensitivity of local sensors, the mass assignments of all pieces of evidence are not exactly the same. Notably, evidence $E_{3}$ is relatively different from other pieces of evidence. Besides, all pieces of evidence correspondingly allocate the biggest support to true proposition $A$. Hence, a reasonable and reliable fusion method should correctly recognize the true proposition $A$.

The fusion of the results of Shafer et al. $[8,16,17,24,25]$ and the proposed fusion algorithm are exhibited in Table 2.

Table 2. Fusion results of different methods with consistent information.

\begin{tabular}{|c|c|c|c|c|c|}
\hline Evidence & Methods & $A$ & $B$ & $C$ & $\Theta$ \\
\hline \multirow{6}{*}{$\begin{array}{l}E_{1} \oplus E_{2} \\
K=0.1970\end{array}$} & DS combination [8] & 0.9863 & 0 & 0.0137 & 0 \\
\hline & Reference [16] & 0.9360 & 0.0008 & 0.0280 & 0.0352 \\
\hline & Reference [17] & 0.9673 & 0.0010 & 0.0317 & 0 \\
\hline & Reference [24] & 0.9673 & 0.0010 & 0.0317 & 0 \\
\hline & Reference [25] & 0.9605 & 0.0011 & 0.0340 & 0.0044 \\
\hline & Proposed & 0.9879 & 0.0004 & 0.0113 & 0.0004 \\
\hline \multirow{6}{*}{$\begin{array}{l}E_{1} \oplus E_{2} \oplus E_{3} \\
K=0.6007\end{array}$} & DS combination [8] & 0.9917 & 0 & 0.0083 & 0.0000 \\
\hline & Reference [16] & 0.6978 & 0.0278 & 0.0708 & 0.2036 \\
\hline & Reference [17] & 0.8525 & 0.0421 & 0.1054 & 0 \\
\hline & Reference [24] & 0.5365 & 0.0246 & 0.0825 & 0.3564 \\
\hline & Reference [25] & 0.6738 & 0.0206 & 0.0883 & 0.2173 \\
\hline & Proposed & 0.9520 & 0.0099 & 0.0318 & 0.0063 \\
\hline \multirow{6}{*}{$\begin{array}{l}E_{1} \oplus E_{2} \oplus E_{3} \oplus E_{4} \\
K=0.6119\end{array}$} & DS combination [8] & 0.9999 & 0 & 0.0001 & 0 \\
\hline & Reference [16] & 0.7454 & 0.0241 & 0.0570 & 0.1735 \\
\hline & Reference [17] & 0.8868 & 0.0337 & 0.0795 & 0 \\
\hline & Reference [24] & 0.5236 & 0.0165 & 0.0606 & 0.3993 \\
\hline & Reference [25] & 0.6671 & 0.0177 & 0.0700 & 0.2452 \\
\hline & Proposed & 0.9736 & 0.0054 & 0.0175 & 0.0035 \\
\hline \multirow{6}{*}{$\begin{array}{l}E_{1} \oplus E_{2} \oplus E_{3} \oplus E_{4} \oplus E_{5} \\
K=0.6507\end{array}$} & DS combination [8] & 1.0000 & 0 & 0 & 0 \\
\hline & Reference [16] & 0.7492 & 0.0260 & 0.0547 & 0.1701 \\
\hline & Reference [17] & 0.8907 & 0.0351 & 0.0742 & 0 \\
\hline & Reference [24] & 0.5799 & 0.0216 & 0.0562 & 0.3423 \\
\hline & Reference [25] & 0.7128 & 0.0236 & 0.0658 & 0.1978 \\
\hline & Proposed & 0.9783 & 0.0050 & 0.0136 & 0.0031 \\
\hline
\end{tabular}

The fusion results of different combination methods in Table 2 reflect that all the above algorithms can accurately recognize true proposition $A$ with different mass assignments. Detailed discussions are shown as follows.

1. DS combination method: According to the fusion results of multiple pieces of evidence using DS combination, we can see that although the DS combination correctly identifies the true proposition $A$, it always totally denies proposition $B$. The absolute negation is harmful to decision 
processing in the multi-sensor system. After checking the DS combination rule, we confirm that the unreasonable fusion results are caused by the "one ballot veto" situation as $m_{1}(B)=0$. Thus, the DS combination is not completely effective when combining consistent pieces of evidence.

2. The work in $[16,17]$ : Based on the optimization of the DS combination rule, Sun et al. $[16,17]$ obtain relatively reliable fusion results. Notably, we can see from the fusion results that Sun et al. [16] assigns mass to $\Theta$ as $m(\Theta) \neq 0$, which indirectly reflects the system uncertainty; simultaneously, Li et al. [17] supports true proposition $A$ with a bigger probability, which improves the fusion accuracy. Hence, Sun et al. $[16,17]$ have their own characteristics when combining consistent information.

3. The work in [24,25]: According to the essential revision of original pieces of evidence, Wei et al. $[24,25]$ achieve correspondingly effective fusion results with an approximate estimation about system uncertainty as $m(\Theta) \neq 0$. Especially when synthesizing multiple pieces of evidence $(N \geq 3)$, Lin et al. [25] identifies true proposition $A$ with bigger support. Therefore, Lin et al. [25] has better fusion performance than Wei et al. [24].

4. The proposed algorithm: No matter how many pieces of evidence are combined, the fusion results of the proposed DS combination method are all rational and effective. Firstly, compared with the DS combination, the proposed algorithm recognizes the correct proposition $A$ and simultaneously assigns certain support to other propositions. Thus, the proposed algorithm guarantees the accurate decision-making condition for the multi-sensor system. Secondly, the proposed algorithm precisely identifies true proposition $A$ with the biggest support compared to other existing modified methods, which embodies its optimal fusion performance when combining consistent information. Last, but not least, the proposed algorithm evaluates the system uncertainty as $m(\Theta) \neq 0$, which further provides a powerful reference for subsequent information processing.

According to the above discussions when combining consistent information, we can conclude that the DS combination has the "one ballot veto" situation, and Sun et al. $[16,17,24,25]$ have different fusion efficiencies with different modifications. Moreover, the proposed DS combination method not only overcomes the "one ballot veto" situation of the DS combination, but also obtains the topgallantfusion results compared with other existing methods. Therefore, the effectiveness and precision of the proposed algorithm are verified.

\subsection{Multi-Sensor Fusion with Conflicting Information}

The experimental analyses above certify that the proposed fusion algorithm based on the improved DS combination method can effectively fuse the relatively consistent information. Next, we will study the algorithm's performance when handling the multi-sensor conflicting information fusion problem.

With the same assumption as Section 6.1 that proposition $A$ is the true object, the conflicting information of the multi-sensor system is shown in Table 3.

Table 3. Mass functions of conflicting information.

\begin{tabular}{cccc}
\hline \multirow{2}{*}{ Sensors } & \multicolumn{3}{c}{ Propositions } \\
\cline { 2 - 4 } & $\boldsymbol{A}$ & $\boldsymbol{B}$ & $\boldsymbol{C}$ \\
\hline$E_{1}: m_{1}(\cdot)$ & 0.90 & 0 & 0.10 \\
$E_{2}: m_{2}(\cdot)$ & 0 & 0.01 & 0.99 \\
$E_{3}: m_{3}(\cdot)$ & 0.50 & 0.20 & 0.30 \\
$E_{4}: m_{4}(\cdot)$ & 0.98 & 0.01 & 0.01 \\
$E_{5}: m_{5}(\cdot)$ & 0.90 & 0.05 & 0.05 \\
\hline
\end{tabular}

Comparing with Table 1, we can see in Table 3 that evidence $E_{2}$ is the conflicting information. Different from other pieces of evidence $E_{1}, E_{3}, E_{4}, E_{5}, E_{2}$ totally denies true proposition $A$. As the 
pieces of multi-sensor information are obviously conflicting, let us see the fusion results of Sun et al. $[8,16,17,24,25]$ and the proposed fusion algorithm in Table 4.

Table 4. Fusion results of different methods with conflicting information.

\begin{tabular}{|c|c|c|c|c|c|}
\hline Evidence & Methods & $A$ & $B$ & $C$ & $\Theta$ \\
\hline \multirow{6}{*}{$\begin{array}{l}E_{1} \oplus E_{2} \\
K=0.9010\end{array}$} & DS combination [8] & 0 & 0 & 1 & 0 \\
\hline & Reference [16] & 0.1647 & 0.0019 & 0.2984 & 0.5350 \\
\hline & Reference [17] & 0.4055 & 0.0045 & 0.5900 & 0 \\
\hline & Reference [24] & 0.4055 & 0.0045 & 0.5900 & 0 \\
\hline & Reference [25] & 0.4317 & 0.0020 & 0.2841 & 0.2822 \\
\hline & Proposed & 0.3911 & 0.0000 & 0.4262 & 0.1827 \\
\hline \multirow{6}{*}{$\begin{array}{l}E_{1} \oplus E_{2} \oplus E_{3} \\
K=0.9703\end{array}$} & DS combination [8] & 0 & 0 & 1 & 0 \\
\hline & Reference [16] & 0.2232 & 0.0335 & 0.2513 & 0.4920 \\
\hline & Reference [17] & 0.4528 & 0.0679 & 0.4793 & 0 \\
\hline & Reference [24] & 0.3502 & 0.0677 & 0.2850 & 0.2971 \\
\hline & Reference [25] & 0.4276 & 0.0535 & 0.2555 & 0.2634 \\
\hline & Proposed & 0.5363 & 0.0074 & 0.3021 & 0.1542 \\
\hline \multirow{6}{*}{$\begin{array}{l}E_{1} \oplus E_{2} \oplus E_{3} \oplus E_{4} \\
K=0.9997\end{array}$} & DS combination [8] & 0 & 0 & 1 & 0 \\
\hline & Reference [16] & 0.3192 & 0.0295 & 0.1881 & 0.4632 \\
\hline & Reference [17] & 0.5948 & 0.0550 & 0.3502 & 0 \\
\hline & Reference [24] & 0.4446 & 0.0523 & 0.1754 & 0.3277 \\
\hline & Reference [25] & 0.5359 & 0.0427 & 0.1884 & 0.2330 \\
\hline & Proposed & 0.9129 & 0.0076 & 0.0755 & 0.0040 \\
\hline \multirow{6}{*}{$\begin{array}{l}E_{1} \oplus E_{2} \oplus E_{3} \oplus E_{4} \oplus E_{5} \\
K=1\end{array}$} & DS combination [8] & 0 & 0 & 1 & 0 \\
\hline & Reference [16] & 0.3781 & 0.0311 & 0.1671 & 0.4237 \\
\hline & Reference [17] & 0.6560 & 0.0540 & 0.2900 & 0 \\
\hline & Reference [24] & 0.5249 & 0.0503 & 0.1366 & 0.2822 \\
\hline & Reference [25] & 0.6077 & 0.0442 & 0.1606 & 0.1875 \\
\hline & Proposed & 0.9505 & 0.0093 & 0.0340 & 0.0062 \\
\hline
\end{tabular}

Different from the fusion results of consistent information in Table 2, the fusion results of conflicting information in Table 4 intuitively illustrate the accuracy and priority of the proposed methods.

1. DS combination method: As the "one ballot veto" paradox caused by $m_{1}(B)=0, m_{2}(A)=0$ exists, the DS combination directly assigns the whole support to wrong proposition $C$, which is apparently discrepant. Comparing with Table 2, the conflicting factors $K$ in Table 4 are generally higher. Hence, the DS combination cannot work anymore in the highly conflicting situations.

2. The works in [16,17]: When synthesizing relatively consistent information, Sun et al. [16] indirectly reflects the system uncertainty as $m(\Theta) \neq 0$. In the highly conflicting situations in Table 4, this advantage is no longer useful. We can seen the fusion results in Table 4 that $m(\Theta) \geq m(H)$, where proposition $H=A, B, C$. Thus, Sun et al. [16] cannot identify the true proposition $A$ anymore, which is unacceptable for the multi-sensor fusion system. At this point, even if Li et al. [17] cannot estimate the system uncertainty, its support assignment to proposition $A$ rises with the increasing of reliable pieces of evidence. However, the growth rate of support assignment to proposition $A$ is slow. Therefore, Sun et al. $[16,17]$ has certain limitations when combining highly conflicting information.

3. The works in [24,25]: Similar to the analyses of Wei et al. [24,25] when synthesizing consistent information, Lin et al. [25] has better fusion performance than Wei et al. [24] as Lin et al. [25] identifies true proposition $A$ with bigger support. However, the growth rate of support 
assignment to true proposition $A$ in [25] is slow, which is not conducive to decision-making in the multi-sensor fusion system.

4. The proposed algorithm: Firstly, when considering the combination of pieces of evidence $E_{1}, E_{2}$, the proposed method does not exhibit good results compared to Li et al. [17,24,25]. Through rational analyses, we find that if there are only two pieces of evidence in the multi-sensor fusion system with high conflicting degree, it is hard for the system to determine which piece of evidence is more reliable. At this point, more sensor information is needed for further combination. This situation indirectly confirms the development demand of the multi-sensor fusion system. Then, with the increasing of pieces of evidence $E_{3}, E_{4}, E_{5}$, the reliability of evidence $E_{1}$ is gradually confirmed. In that case, we can see in Table 4 that the proposed algorithm rapidly increases its support to true proposition $A$, which is profitable for the multi-sensor fusion system. It is worth mentioning that, when the fusion pieces of evidence are greater than two, the proposed algorithm can identify the true proposition $A$ with the largest support compared with other modified methods. Thus, the great fusion performance and fast convergence ability of the proposed algorithm is proven.

As previously mentioned, when combing conflicting information, the DS combination will encounter the "one ballot veto" paradox, Sun et al. [16] cannot identify the true proposition $A$ anymore, and Li et al. $[17,24,25]$ obtains reasonable fusion results with different accuracies. Besides, the proposed DS combination method not only overcomes the "one ballot veto" paradox of the DS combination, but also obtains the optimal fusion results compared with other methods when $N \geq 3$. Accordingly, the reliability and robustness of the proposed algorithm are certified.

On the basis of the above two experimental analyses, the proposed algorithm has a prominent advantage and outstanding priority compared with the other five fusion methods.

\section{Conclusions}

Information fusion has become a mainstream trend in various multi-sensor systems. Due to the limited sensor sensitivity, the lack of a priori knowledge of the environment and wrong information preprocessing, uncertainty inevitably occurs in the multi-sensor fusion system. DS theory, as an effective uncertainty reasoning method, is applied to establish the multi-sensor conflicting fusion system in this paper. When system uncertainty becomes huge, DS theory will obtain irrational fusion results in highly conflicting situations. After the discussions of conflicting sources and existing modifications, we put forward a novel DS combination method for conflicting information fusion in this paper. The potentially conflicting pieces of evidence are firstly revised respectively by the modified Minkowski distance function and the betting-commitment distance function and then combined by an improved conflicting redistribution strategy based on locally-conflicting analyses. Via the comprehensive processing of conflicting pieces of evidence, reasonable and effective fusion results can be obtained. Finally, the experimental results and analyses illustrate the validity and accuracy of the proposed algorithm when combining consistent information and prove its effectiveness and priority when combining conflicting information. Accordingly, the proposed algorithm has certain significance for the multi-sensor fusion system.

Acknowledgments: The paper is funded by the National key research and development program of China (No. 2016YFF0102806) and the Ph.D. Student Research and Innovation Fund of the Fundamental Research Funds for the Central Universities of China (No. HEUGIP201708). Moreover, this work is supported by the National Natural Science Foundation of China (No. 61701134) and the Natural Science Foundation of Heilongjiang Province, China (No. F2017004).

Author Contributions: Jie Chen and Fang Ye conceived of the concept and performed the research. Jie Chen conducted the experiments to evaluate the performance of the proposed multi-sensor conflicting information fusion algorithm based on the improved DS combination method. Tao Jiang and Yuan Tian reviewed the manuscript. All authors have read and approved the final manuscript.

Conflicts of Interest: The authors declare no conflict of interest. 


\section{References}

1. Raol, J.R. Multi-source multi-sensor information fusion. Sadhana 2004, 29, 143-144.

2. Li, T.; Corchado, J.M.; Sun, S. Clustering for filtering: Multi-object detection and estimation using multiple/massive sensors. Inf. Sci. 2017, 388, 172-190.

3. Caballero-Aguila, R.; Hermosocarazo, A.; Linaresperez, J. New distributed fusion filtering algorithm based on covariances over sensor networks with random packet dropouts. Int. J. Syst. Sci. 2017, 48, 1805-1817.

4. Chu, J.F.; Liu, X.W.; Gong, Z.W. Two Decision Making Models Based on Newly Defined Additively Consistent Intuitionistic Preference Relation. In Proceedings of the IEEE International Conference on Fuzzy Systems, Istanbul Turkey, Istanbul, Turkey, 2-5 August 2015.

5. Deng, X.; Jiang, W. Fuzzy Risk Evaluation in Failure Mode and Effects Analysis Using a D Numbers Based Multi-Sensor Information Fusion Method. Sensors 2017, 17, 2086.

6. Xu, W.; Li, M.; Wang, X. Information Fusion Based on Information Entropy in Fuzzy Multi-source Incomplete Information System. Int. J. Fuzzy Syst. 2017, 19, 1200-1216.

7. Dempster, A.P. Upper and lower probabilities induced by a multi-valued mapping. Ann. Math. Stat. $1967,38,325-329$.

8. Shafer, G. A Mathematical Theory of Evidence; Princeton University Press: Princeton, NJ, USA, 1976.

9. Jiang, W.; Zhuang, M.; Xie, C. A Reliability-Based Method to Sensor Data Fusion. Sensors 2017, $17,1575$.

10. Bhatt, D.; Babu, S.R.; Chudgar, H.S. A novel approach towards utilizing Dempster Shafer fusion theory to enhance WiFi positioning system accuracy. Pervasive Mob. Comput. 2017, 37, 115-123.

11. Yin, L.; Zheng, H.; Bian, T.; Deng, Y. An evidential link prediction method and link predictability based on Shannon entropy. Phys. A Stat. Mech. Appl. 2017, 482, 699-712.

12. Ye, F.; Chen, J.; Li, Y.B. Improvement of DS Evidence Theory for Multi-Sensor Conflicting Information. Symmetry 2017, 9, 69.

13. Zadeh, L.A. Book Review: A Mathematical Theory of Evidence. Bull. Am. Math. Soc 1984, 10, 235-247.

14. Smets, P. The combination of evidence in the transferable belief model. IEEE Trans. Pattern Anal. Mach. Intell. 1990, 12, 447-458.

15. Yager, R.R. On the aggregation of prioritized belief structures. Pattern Anal. Mach. Intell. 1996, 26, 708-717.

16. Sun, Q.; Ye, X.Q.; Gu, W.K. A new combination rules of evidence theory. Chin. J. Electron. 2000, 28, 117-119.

17. Li, B.C.; Huang, J.; Yin, H.J. Two Efficient Combination Rules for Conflicting Belief Functions. In Proceedings of the International Conference on Artificial Intelligence and Computational Intelligence, Shanghai, China, 7-8 November 2009; Volume 61, pp. 421-426.

18. Zhang, B.; Lu, H.Z. Combination method of conflict evidence in multi-sensor automatic target recognition. Syst. Eng. Electron. 2006, 28, 857-860.

19. Zhang, S.Y.; Pan, Q.; Zhang, H. A New Kind of Combination Rule of Evidence Theory. Control Decis. 2000, 15, 540-544.

20. Murphy, C.K. Combining belief functions when evidence conflicts. Decis. Support Syst. 2000, 29, 1-9.

21. Horiuchi, T. Decision rule for pattern classification by integrating interval feature values. IEEE Trans. Pattern Anal. Mach. Intell. 1998, 20, 440-448.

22. Deng, Y.; She, W.K.; Zhu, Z.F. Efficient combination approach of conflict evidence. J. Infrared Millim. Waves 2004, 23, 27-32.

23. Guo, H.W.; Shi, W.K.; Liu, Q.K.; Deng, Y. A new combination rule of evidence. J. Shanghai Jiao-Tong Univ. 2007, 40, 1895-1900.

24. Wei, Y.C. An improved D-S evidence combination method based on K-L distance. Telecommun. Eng. 2011, 27, 255-257.

25. Lin, Y.; Wang, C.; Ma, C.G. A new combination method for multisensor conflict information. J. Supercomput. 2016, 72, 2874-2890.

26. Deng, Y.; Chan, F.T. A new fuzzy dempster MCDM method and its application in supplier selection. Expert Syst. Appl. 2011, 38, 9854-9861.

27. Beynon, M.; Cosker, D.; Marshall, D. An expert system for multi-criteria decision making using Dempster Shafer theory. Expert Syst. Appl. 2001, 20, 357-367.

28. Dymova, L.; Sevastjanov, L. An interpretation of intuitionistic fuzzy sets in terms of evidence theory: Decision making aspect. Knowl.-Based Syst. 2010, 23, 772-782. 
29. Kang, J.; Gu, Y.B.; Li, Y.B. Multi-sensor information fusion algorithm based on DS evidence theory. J. Chin. Inert. Technol. 2012, 20, 670-673.

30. Zeng, R.; Zhang, L.; Mei, J. Fault detection in an engine by fusion information from multivibration sensors. Int. J. Distrib. Sens. Netw. 2017, 13, doi:10.1177/1550147717719057.

31. Fan, X.F.; Zuo, M.J. Fault diagnosis of machines based on D-S evidence theory. Part 2: Application of the improved D-S evidence theory in gearbox fault diagnosis. Pattern Recognit. Lett. 2006, 27, 377-385.

32. Dezert, J. Autonomous navigation with uncertain reference points using the PDAF. In Multitarget-Multisensor Tracking; Bar-Shalom, Y., Ed.; Artech House: Norwood, MA, USA, 2016; Volume 2, pp. 271-327.

33. Baiocchi, V.; Bianchi, A.; Maddaluno, C.; Vidale, M. Pansharpening techniques to detect mass monument damaging in IRAQ. Int. Arch. Photogr. Remote Sens. Spat. Inf. Sci. 2017, XLII-5/W1, 121-126.

34. Merigo, J.M.; Casanovas, M. A New Minkowski Distance Based on Induced Aggregation Operators. Int. J. Comput. Intell. Syst. 2011, 4, 123-133.

35. Smets, P. Decision making in the TBM: The necessity of the pignistic transformation. Int. J. Approx. Reason. 2005, 38, 133-147.

36. Han, D.Q.; Deng, Y.; Han, C.Z. Some notes on betting commitment distance in evidence theory. Sci. China 2012, 55, 558-565.

(C) 2017 by the authors. Licensee MDPI, Basel, Switzerland. This article is an open access article distributed under the terms and conditions of the Creative Commons Attribution (CC BY) license (http://creativecommons.org/licenses/by/4.0/). 